\title{
Artificially Created Reentry Circuit by Laser Irradiation Causes Atrial Tachycardia to Persist in Murine Atria
}

\author{
Shunpei Horii, MD; Hirotaka Yada, MD; Kei Ito, MD; Kazuhiro Tsujita; \\ Ayumu Osaki, MD; Kazuki Kagami, MD; Atsushi Sato, MD; Toyokazu Kimura, MD; \\ Risako Yasuda, MD; Takumi Toya, MD; Takayuki Namba, MD; Yuji Nagatomo, MD; \\ Yasuo Ido, MD; Koji Miyazaki, MD; Nobuyuki Masaki, MD; \\ Miya Ishihara, PhD; Bonpei Takase, MD; Takeshi Adachi, MD
}

Background: There is a gradual progression from paroxysmal to persistent atrial fibrillation (AF) in humans. To elucidate the mechanism involved, the creation of an artificial atrial substrate to persist AF in mice was attempted.

\begin{abstract}
Methods and Results: This study used wild type (WT) mice, but it is difficult to induce AF in them. A novel antegrade perfusion method from the left ventricle (LV) to enlarge both atria for artificial atrial modification was proposed in this study. Short duration AF was induced by burst pacing under this method. Optical mapping analysis revealed non-sustained focal type and meandering spiral reentrants after short duration AF. A tiny artificial substrate $(\sim 1.2 \mathrm{~mm}$ in diameter) was added in by laser irradiation to create a critical atrial arrhythmogenic substrate. Burst pacing was performed in a non-laser group $(n=8)$, a circular-shape laser group ( $n=8)$, and a wedge-shaped dent laser group $(n=8)$. We defined AF and atrial tachycardia (AT) as atrial arrhythmia (AA). Long-lasting AA was defined as lasting for $\geq 30$ min. Long-lasting $A A$ was observed in $0 / 8,0 / 8$, and $6 / 8$ (75\%) mice in each group. Optical mapping analysis revealed that the mechanism was AT with a stationary rotor around the irradiated margin.
\end{abstract}

Conclusions: Regrettably, this study failed to reproduce persistent AF, but succeeded in creating an arrhythmic substrate that causes sustained AT in WT mice.

Key Words: Atrial fibrillation; Artificial reentry; Atrial tachycardia; Laser irradiation

A trial fibrillation (AF) is the most common arrhythmic disease and humans are known to be susceptible it to as they age. ${ }^{1}$ Over a natural course, AF starts in a paroxysmal form and progresses to a persistent form with the passage of time. ${ }^{2}$ AF can be maintained by reentry and/or rapid focal ectopic firing, and AF-related electrical remodeling favors the development of a functional and structural reentry substate. ${ }^{3}$ However, it is difficult to clearly distinguish the difference in electrical characteristics between paroxysmal AF and persistent AF. Progression from paroxysmal AF to persistent AF increases thrombotic events and mortality rates. ${ }^{4}$ Although premature catheter ablation therapy may prevent the development of AF, $\mathbf{5}$ the STAR AF II trial revealed that the only effective proven strategy for persistent AF ablation was pulmonary vein isolation, and that the ablation success rate in persistent AF was insufficient as compared

\section{Editorial p330}

with paroxysmal AF. ${ }^{6}$ We assumed that elucidation of mechanisms of AF progression would be important to prevent and treat persistent AF.

In mice, it was possible to induce AF by electrical burst stimulation; ${ }^{7}$ however, AF lasted for only a short duration in WT mice. Extension of AF duration to around several hours was only reported for genetically engineered mice (metabolic disorder mice 8 and ion channel abnormal mice $\left.{ }^{9,10}\right)$, and increased fibrosis of the atrial muscle was observed in these mice. The above studies suggested that atrial substrates (structural remodeling11-13 and electrical remodeling ${ }^{14-16}$ ) were involved in the progression from short-duration AF to long-lasting AF.

We hypothesized that a critical atrial substrate would

Received January 28, 2021; revised manuscript received July 14, 2021; accepted July 18, 2021; J-STAGE Advance Publication released online September 17, 2021 Time for primary review: 17 days

Department of Cardiology (S.H., K.I., A.O., K.K., A.S., T.K., R.Y., T.T., T.N., Y.N., Y.I., T.A.), Department of Medical Engineering (K.T., M.I.), Department of Intensive Care Medicine (N.M., B.T.), National Defense Medical College, Tokorozawa; Department of Cardiology, International University of Health and Welfare Mita Hospital, Tokyo (H.Y.); and Department of Comprehensive Internal Medicine, Tokai University Hachioji Hospital, Hachioji (K.M.), Japan

Mailing address: Hirotaka Yada, MD, Department of Cardiology, International University of Health and Welfare Mita Hospital, 1-4-3 Mita, Minato-ku, Tokyo 108-8329, Japan. E-mail: hyada@iuhw.ac.jp

All rights are reserved to the Japanese Circulation Society. For permissions, please e-mail: cj@j-circ.or.jp

ISSN-1346-9843 


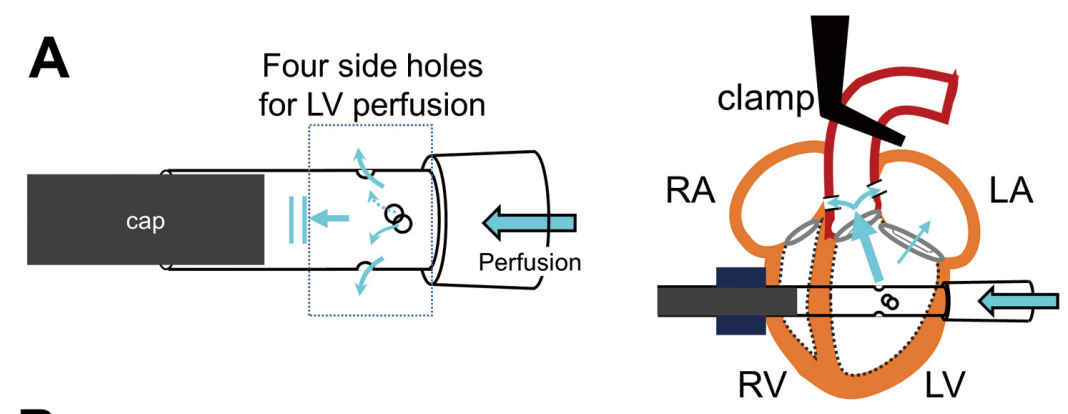

B
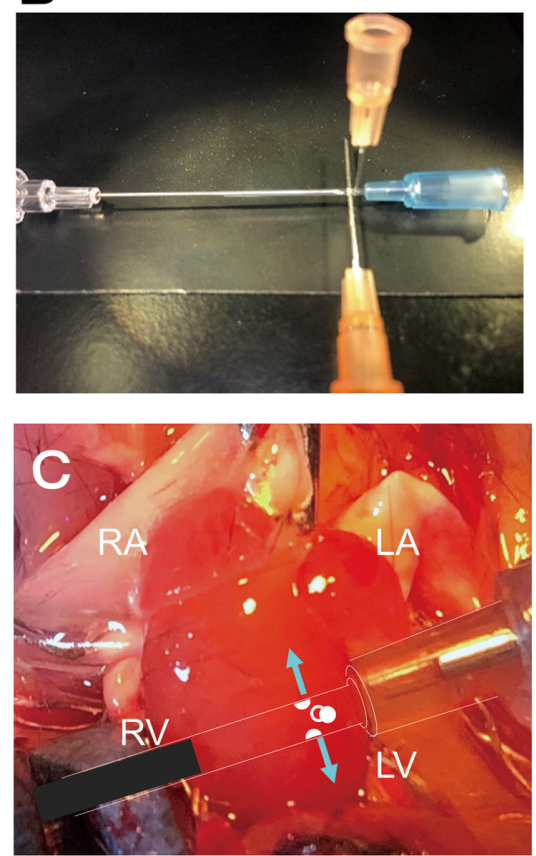
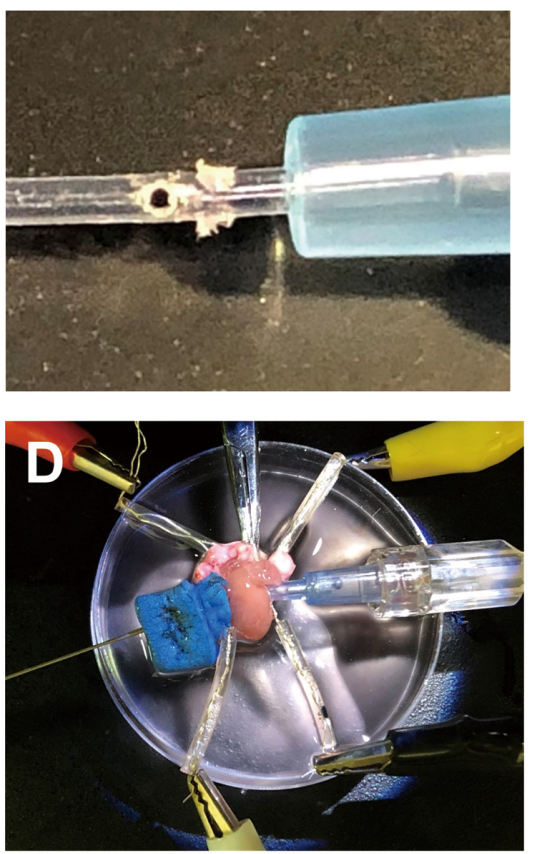

Figure 1. (A) Schema of an antegrade perfusion method for assessment of atrial electrophysiology. A catheter with 4 side holes was inserted from the left ventricle (LV) to the right ventricle $(\mathrm{RV})$. The distal side was fixed by a hard-type sponge and the distal tip was occluded by a puncture needle. The coronary artery was perfused in the antegrade direction from the LV, as shown in the schema. (B) Twenty-two needle gauge perfusion venous catheter with 4 side holes for antegrade perfusion. Side holes were created by using a 25 needle gauge vertically. The position of the side holes was adjusted according to the size of the heart. (C) Descending aorta, IVC, and lower lobe of both lungs were cut before LV cannulation. Ascending aorta including SVC was clamped after LV perfusion. Distal side of the clamp was cut and moved to a 6-cm dish with an electrode. (D) Overall view after antegrade perfusion. Electrode placed in each position (red: right upper hilum, yellow: left upper hilum, white: RV, black: LV). The perfusate stored in the dish can be reused for LV perfusion. promote the transition from short-duration to long-lasting AF; however, it would be difficult to distinguish the critical substrate in genetically engineered mouse models, because of their specialized electrical and structural remodeling compared with WT mice. Therefore, we used WT mice in which long-lasting AF cannot usually be induced, thinking that if we could generate a substrate that sustained AF artificially in WT mice, it could be recognized as one of the critical substrates for long-lasting AF in mice. To identify a critical substrate, we used laser irradiation to create an artificial substrate on the atrium. The severity of damage, shape, and size of the substrate in atrial tissue can be controlled with adjusted laser irradiation. Laser irradiation enables us to generate a comparatively free-form substrate. The retrograde coronary perfusion using the classical Langendorff perfusion system sometimes caused the collapse of the atrium, and therefore, laser irradiation in front of the atrium was sometimes impossible (Supplementary Figure A-E). Therefore, we devised a novel Langendorfffree method with reference to a past report of murine cardiac myocyte isolation. ${ }^{17} \mathrm{We}$ performed Tyrode perfusion from the LV to prevent the atrium from collapsing. This method made laser irradiation on the mouse atrium easier and more stable. Two types of arrhythmias, short duration
AF and long-lasting AT, were induced in the laser irradiation experiment. The distinction between the 2 was difficult on electrocardiogram (ECG) and was only distinguished by optical mapping analysis. Therefore, we collectively defined AF and AT as AA in this study.

\section{Methods}

\section{An Antegrade Perfusion Method}

A schematic diagram of an antegrade perfusion is shown in Figure 1A. Mice (C57BL/6 male mice, aged 12 weeks) were anesthetized with a mixture of 3 anesthetics (medetomidine hydrochloride $0.3 \mathrm{mg} / \mathrm{kg}+$ midazolam $4 \mathrm{mg} / \mathrm{kg}$ + butorphanol tartrate $5 \mathrm{mg} / \mathrm{kg}$ ) and heparin (100 units) by intraperitoneal injection. A 22-gauge peripheral intravenous catheter with 4 side holes (Figure 1B) was inserted from the LV to the right ventricle (RV) and the tip of the cannula was closed (Figure 1C). Oxygenation was performed $\left(95 \% \quad \mathrm{O}_{2}, \quad 5 \% \quad \mathrm{CO}_{2}\right)$ and Tyrode solution $\left(128.2 \mathrm{mmol} / \mathrm{L} \mathrm{NaCl}, 1.3 \mathrm{mmol} / \mathrm{L} \mathrm{CaCl}_{2}\left(2 \mathrm{H}_{2} \mathrm{O}\right), 4.7 \mathrm{mmol} / \mathrm{L}\right.$ $\mathrm{KCl}, 1.05 \mathrm{mmol} / \mathrm{L} \mathrm{MgCl}_{2}\left(6 \mathrm{H}_{2} \mathrm{O}\right), 1.19 \mathrm{mmol} / \mathrm{L} \mathrm{NaH}_{2} \mathrm{PO}_{4}$, $20 \mathrm{mmol} / \mathrm{L} \mathrm{NaHCO}, 11.1 \mathrm{mmol} / \mathrm{L}$ D-Glucose in deionized water, $\mathrm{pH}=7.35 \pm 0.05$ ) at $37^{\circ} \mathrm{C}$ was introduced. Next, the descending aorta / inferior vena cava was cut to prevent 
perfusion fluid stagnation and blood was replaced with Tyrode solution. The whole heart was cut at the distal side of the clamp and moved on to a 6-cm dish (Figure 1D). We can reuse the solution that was collected in the dish. To avoid excessive pressure on the LA, the right and left lower lobe of the lung were excised and the peristaltic pumping perfusion pressure was maintained at $110-120 \mathrm{mmHg}$, and both atrial and ventricular pressures were measured (Figure 2A). Chamber pressure on peristaltic pumping was measured, as shown in Supplementary Figure. When observing by ECG or optical mapping method, 2, 3-butanedione monoxime (BDM) $(10 \mathrm{mmol} / \mathrm{L})$ was mixed with Tyrode solution to suppress the motion artifact due to heartbeat. ${ }^{18}$ Ventricular electrodes were placed on both sides of the ventricle, and an atrial electrode was placed on the hilum of the upper lung lobe to record ECG and stimulation (Figure 1D). Furthermore, when observing optical mapping or laser irradiation, drip infusion was used to reduce any slight atrial movement caused by peristaltic pumping.

\section{Optical Mapping of Mouse Hearts Under an Antegrade Perfusion Method}

Optical mapping was performed according to previously reported protocols. ${ }^{19}$ Hearts were stained with voltagesensitive dye, RH237 stock solution $(1.25 \mathrm{mg} / \mathrm{mL}$ solution in dimethyl sulfoxide [DMSO, Sigma]). Tyrode solution containing RH237 is injected almost 10 min for dyeing. The light from a filtered light emitting diode (524-574 nm) was reflected onto the epicardial fluorescence via a dichroic mirror. Fluorescence images were acquired through a long pass filter $(>700 \mathrm{~nm})$ using a CMOS camera (MICAM Ultima, Brainvision, Tokyo, Japan) with a $1 \times 1 \mathrm{~cm}$ field of view. All optical signals were captured at $1,000 \mathrm{~Hz}$ and processed using BV analysis software (Brain Vision, Tokyo, Japan). To analyze the activated patterns and areas, the activation and phase map function of the BV analysis software was used. In phase singularity space-time plot analysis, phase singularity points ${ }^{20}$ were investigated at intervals of approximately $15-30 \mathrm{~ms}$.

\section{Electrical Stimulation for AA Induction}

We tried 3 types of electrical stimulation from the RA, LA, and PV for AA induction. Electrical stimulation from the anterior side of right atrium (RA) and left atrium (LA) was perfomed by using bipolar stimulation (1.1 F EPR-800 catheter; Millar, TX, USA). In PV stimulation, looped silver electrodes with an insulator grabbed hilum from the upper lobe of both lungs (Figure 1D, red and yellow electrodes). We made the electrode loop smaller to grasp the pulmonary vein stably and paced. We applied $30 \mathrm{~V}$ and $50 \mathrm{~Hz}$ burst pacing for AA induction for each type of stimulation. Stimulation duration was fixed at $\sim 500 \mathrm{~ms}$. The stimulaiton was delivered 5 times in the AA induction test and the longest AA duration was adopted. The AA induction rate and duration was preferable in PV stimulation. We used PV stimulation and performed stimulation 3 times during 1 laser irradiation session in a laser experiment.

\section{Artificial Substrate Created by Laser Irradiation in Mouse Hearts}

As shown in Figure 6A, light from a laser source was guided using a mirror. After focusing on the atrial surface, it was defocused to adjust for the size of laser irradiation. The laser source used was a Q-switched Nd: YAG laser
(Minilite II; Continuum, San Jose, CA, USA) device. The wavelength was $532 \mathrm{~nm}$, the pulse width (time of one irradiation) was $3-5 \mathrm{nsec}$, and the irradiation frequency was $10 \mathrm{~Hz}$. The irradiation conditions and evaluation methods for the laser light were as follows:

(1) Irradiation range: diameter $1.2 \mathrm{~mm}$.

(2) A wedge-shaped dent pattern was created by irradiation using the laser after placing a wedge-shaped mask above the atrium at $2-3 \mathrm{~mm}$.

(3) Energy amount per irradiation: the energy amount was increased step-by-step to $2 \mathrm{~mJ} /$ times, $4 \mathrm{~mJ} /$ times, and $8 \mathrm{~mJ} /$ times.

(4) Irradiation time and irradiation frequency: 3 sets with $2 \mathrm{~mJ}$ for $60 \mathrm{~s}$ (600 times irradiation), 3 sets with $4 \mathrm{~mJ}$ for $60 \mathrm{~s}$ (600 times irradiation), 3 sets with $8 \mathrm{~mJ}$ for $60 \mathrm{~s}(600$ times irradiation), 3 sets with $8 \mathrm{~mJ}$ for $120 \mathrm{~s}$ (1,200 times irradiation), 3 sets with $8 \mathrm{~mJ}$ for $240 \mathrm{~s}$ (2,400 times irradiation)

Stimulation was performed 3-fold for each set of laser irradiation.

\section{NADH Autofluorescence Imaging}

Epicardial NADH fluorescence of hearts under an antegrade perfusion was observed, as previously described, with some modifications. ${ }^{\mathbf{2 0 , 2 1}} \mathrm{NADH}$ has an absorption wavelength band of $320-380 \mathrm{~nm}$ and autofluorescence occurs in a wavelength band of $420-480 \mathrm{~nm} .{ }^{22}$ Our observation system was constructed in consideration of this absorption band and fluorescence wavelength band. Irradiation with excitation light was conducted from obliquely above the mouse hearts under an antegrade perfusion for $15 \mathrm{~s}$. The excitation light was generated by a xenon light source (MAX 302; Asahi Spectra Co., Tokyo, Japan) and passed through a bandpass filter (EM $365 \pm 5 \mathrm{~nm}$ ). Images of autofluorescence generated by the excitation light were taken using a camera (D750 Nikon; Tokyo, Japan) equipped with a barrier filter ( $380 \mathrm{~nm}, \mathrm{SCF}$ -

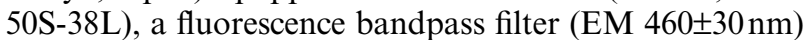
and a macro lens (Micro-Nikkor, $\mathrm{f}=105 \mathrm{~mm}$ ). The intensity distribution of autofluorescence images was analyzed using Image $\mathbf{J}$ (National Institutes of Health).

\section{Stastical Analysis}

All measurements were expressed as mean \pm SD. One-way repeated-measures ANOVA with Bonferroni were used for multiple comparisons when the data were normally distributed. Otherwise, the Wilcoxon signed-ranks test was used for 2-group comparisons. All of the analyses were conducted using $\mathrm{R}$ version 3.6.1 (2019). Values of $\mathrm{P}<0.05$ were considered to be significant.

\section{Results}

\section{Electrical Activation During Sinus Rhythm by Optical Mapping Using a Novel Antegrade Perfusion}

To observe epicardial action potentials of the murine atrium by optical mapping, a Langendorff-based approach, in which the atrium is stained with voltage-sensitive dyes, is normally used. We devised a novel antegrade perfusion method for laser irradiation from the front of the atrium. Tyrode was perfused in an antegrade perfusion manner from the LV to the ascending aorta and coronary artery using a 22-gauge peripheral intravenous catheter with 4 side holes (Figure 1). Aortic (Ao) pressure $(75.4 \pm 6.4 / 53.5 \pm 9.1 \mathrm{mmHg}$, $\mathrm{n}=8$ ) was approximately $30-40 \mathrm{mmHg}$ lower than peristal- 


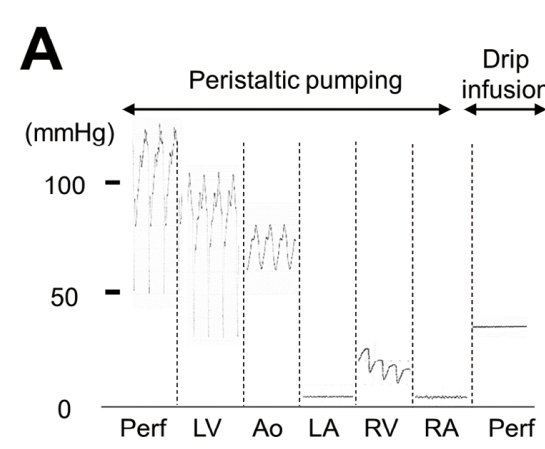

Chamber pressure during optical mapping dye staining (peristaltic pumping) $(n=8)$

\begin{tabular}{|c|c|}
\hline $\begin{array}{c}\text { Perfusion } \\
\text { pressure }\end{array}$ & $116 \pm 9.9 / 69.5 \pm 7.2 \mathrm{mmHg}$ \\
\hline $\mathrm{LV}$ & $95.1 \pm 6.4 / 63.3 \pm 5.4 \mathrm{mmHg}$ \\
\hline Aorta & $75.4 \pm 8.1 / 53.5 \pm 9.1 \mathrm{mmHg}$ \\
\hline $\mathrm{LA}$ & $5.4 \pm 5.0 \mathrm{mmHg}$ \\
\hline RV & $22 \pm 7.4 / 11.8 \pm 5.1 \mathrm{mmHg}$ \\
\hline RA & $2.8 \pm 1.5 \mathrm{mmHg}$ \\
\hline
\end{tabular}

Constant perfusion pressure during laser irradiation (Drip infusion) $\quad(n=8)$

\begin{tabular}{|l|l|}
\hline $\begin{array}{c}\text { Perfusion } \\
\text { pressure }\end{array}$ & $38.8 \pm 3.2 \mathrm{mmHg}$ \\
\hline
\end{tabular}
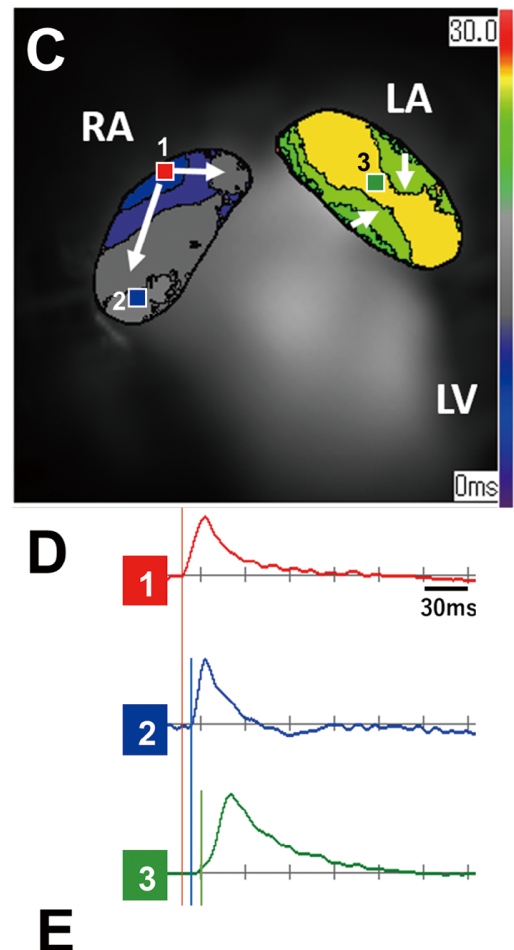

E

\begin{tabular}{|c|c|c|}
\hline $\begin{array}{c}\sim 1^{\text {st }} \text { degree } \\
\text { AV block }\end{array}$ & $\begin{array}{c}2^{\text {nd }} \text { degree } \\
\text { AV block }\end{array}$ & $\begin{array}{c}\text { Complete } \\
\text { AV block }\end{array}$ \\
\hline $3 / 8(38 \%)$ & $3 / 8(38 \%)$ & $2 / 8(25 \%)$ \\
\hline \hline
\end{tabular}
B Action potential duration (APD) and
conduction velocity (CV)

\begin{tabular}{|c|c|c|}
\hline & $\mathrm{APD}_{80}(\mathrm{msec})$ & $\mathrm{CV}(\mathrm{cm} / \mathrm{s})$ \\
\hline RA & $24.9 \pm 2.6$ & $46.1 \pm 7.7$ \\
\hline LA & $23.1 \pm 4.2$ & $41.8 \pm 9.3$ \\
\hline LV & $47.1 \pm 3.5$ & $69.5 \pm 7.7$ \\
\hline
\end{tabular}

Normal
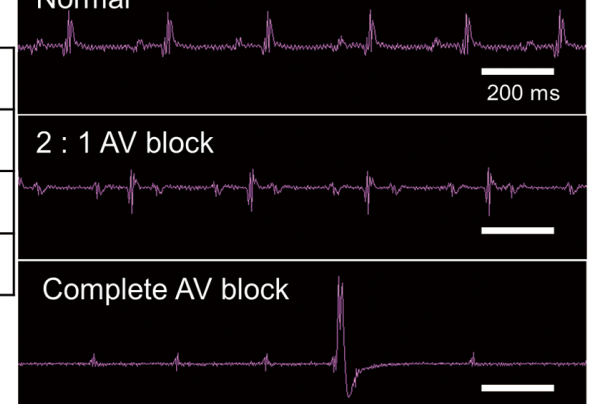

Figure 2. (A) Chamber pressure during peristaltic pumping from the left ventricle (LV). Detailed methods of pressure measurement is shown in Supplementary Figure. The fluctuation in the right ventricle (RV) pressure is considered due to the leakage from the $\mathrm{LV}$ to the RV around the perfusion catheter. The peristaltic pumping was switched to a constant drip infusion to reduce atrial movement as much as possible under enforcement of laser irradiation and optical mapping study. Perf, perfusion. (B) Action potential duration (APD) 80 and conduction velocity (CV) at $200 \mathrm{~ms}$ burst pacing. (C) Optical activation map during sinus rhythm in a mouse heart under an antegrade perfusion method. (D) Optical action potentials corresponding to points 1-3 in the right atrium (RA) and the left atrium (LA). Activation originates from the sinus node in the upper RA, leading to inferior RA and LA. (E) Atrioventricular block of more than a second degree was observed in 5/8 (63\%), which was considered to be caused by the damage of the atrioventricular (AV) node or His bundle.

tic perfusion pressure, and we assumed that coronary artery pressure would be sufficient for staining of fluorescence dye (Figure 2A). Mean LA pressure $(5.4 \pm 5.0 \mathrm{mmHg}$, $\mathrm{n}=8$ ) was appropriate and excessive pressure was not applied. Atrial area of the anterior surface was larger during LV perfusion compared with during Ao perfusion (RA $7.6 \pm 0.7$ vs. $6.1 \pm 0.6 \mathrm{~mm}^{2}$, LA $7.8 \pm 0.5$ vs. $4.9 \pm 1.0 \mathrm{~mm}^{2}, \mathrm{n}=8$, $\mathrm{P}<0.001$, Supplementary Figure $\mathbf{C}-\mathbf{E})$. Action potential duration and conduction velocity at $200 \mathrm{~ms}$ pacing are shown in Figure 2B. We were able to confirm normal atrial activation from the upper RA to the LA using our novel antegrade perfusion method (Figure 2C,D). In this method, ventricular septal puncture caused atrioventricular (AV) block. Second degree and complete AV block were recognized in $3 / 8(38 \%)$ and $2 / 8(25 \%)$ of mouse hearts under an antegrade perfusion method.

Induction and Electrical Characteristics of AF in WT Mice

The duration of AF was significantly longer in PV stimulation $(13.5 \pm 13.8 \mathrm{~s}, \mathrm{n}=8)$ compared to RA stimulation 

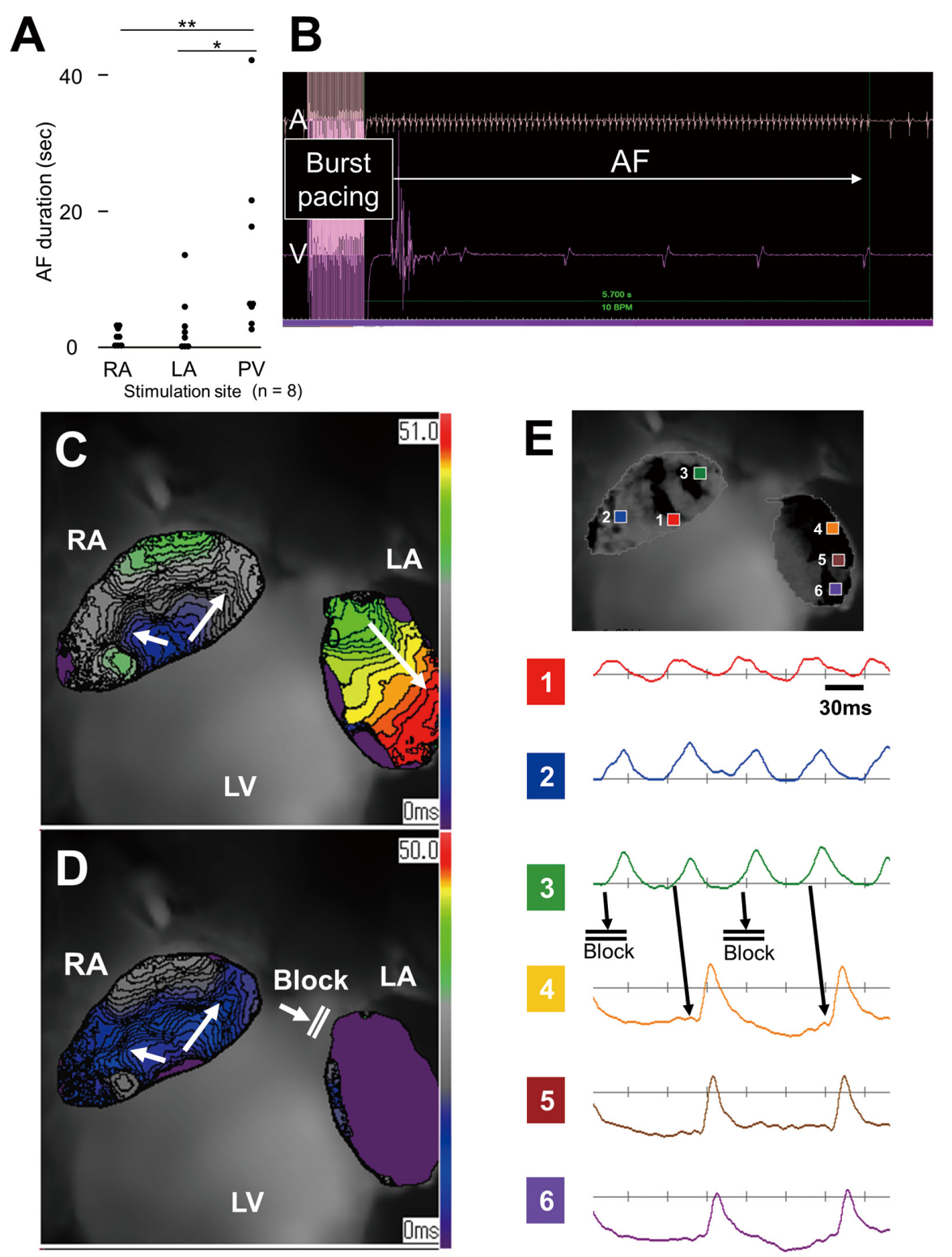

Figure 3. (A) Induction of atrial fibrillation (AF) was performed from the front of the right atrium (RA), left atrium (LA), and PV (from both hilum). Short duration high-frequency burst pacing $(500 \mathrm{~ms}, 50 \mathrm{~Hz}$ and $30 \mathrm{~V})$ was delivered. The AF duration was longer in the PV stimulation group (13.5 $\pm 13.8 \mathrm{~s}, \mathrm{n}=8)$ compared with the RA group $(1.4 \pm 1.3 \mathrm{~s}, \mathrm{n}=8)$ and the LA group $(3.2 \pm 4.8 \mathrm{~s}, \mathrm{n}=8)$. ${ }^{*} \mathrm{P}<0.05$, ${ }^{\star *} P<0.01$. (B) AF was successfully induced from $P V$ pacing under an antegrade perfusion. (C,D) Optical activation mapping of shortduration ectopic focal AF in wild-type (WT) mice. After ectopic firing from the peri-tricuspid valve of the anterior RA, the impulse wave was conducted to the LA once every 2 times and blocked the other time. (E) Optical action potentials (points 1-6) on the conduction path of ectopic firing. There is a 2:1 block of the impulse conduction between points 3 and 4 (between the RA and the LA).

$(1.4 \pm 1.3 \mathrm{~s}, \mathrm{n}=8$, vs. $\mathrm{PV}, \mathrm{P}<0.01)$ and $\mathrm{LA}$ stimulation $(3.3 \pm 4.8 \mathrm{~s}, \mathrm{n}=8$, vs. $\mathrm{PV}, \mathrm{P}<0.05)$ (Figure 3A,B). We adopted PV stimulation based on these results.

We observed the electrical dynamics of short-duration AF using optical mapping. Two representative types of $\mathrm{AF}^{23}$ were observed during short-duration AF in optical mapping analysis. One mechanism was an ectopic focus type, and ectopic foci were outside of the sinus node (SN) produced by abnormal automaticity activities. ${ }^{20}$ Figure 3C,D, and $\mathbf{E}$ show the ectopic focus type AF. Ectopic focal excitation from the right anterior atrium near the tricuspid valve spreads throughout the RA and is transmitted to the LA. Tachycardia cycle length was approximately 45-50 ms. Conduction to the LA was blocked once every 2 times. Another mechanism was spiral wave type, and spiral activation wave rotates around a spiral wave center. Figure 4 shows optical phase map of the rare left-right reverse rotation spiral wave AF. Multiple, simultaneous spiral waves were identified in both the RA and LA. Tachycardia cycle length was approximately $40-45 \mathrm{~ms}$ in the RA and $45-50 \mathrm{~ms}$ in the LA. Activation waves independently rotate counterclockwise in the RA, and clock- 


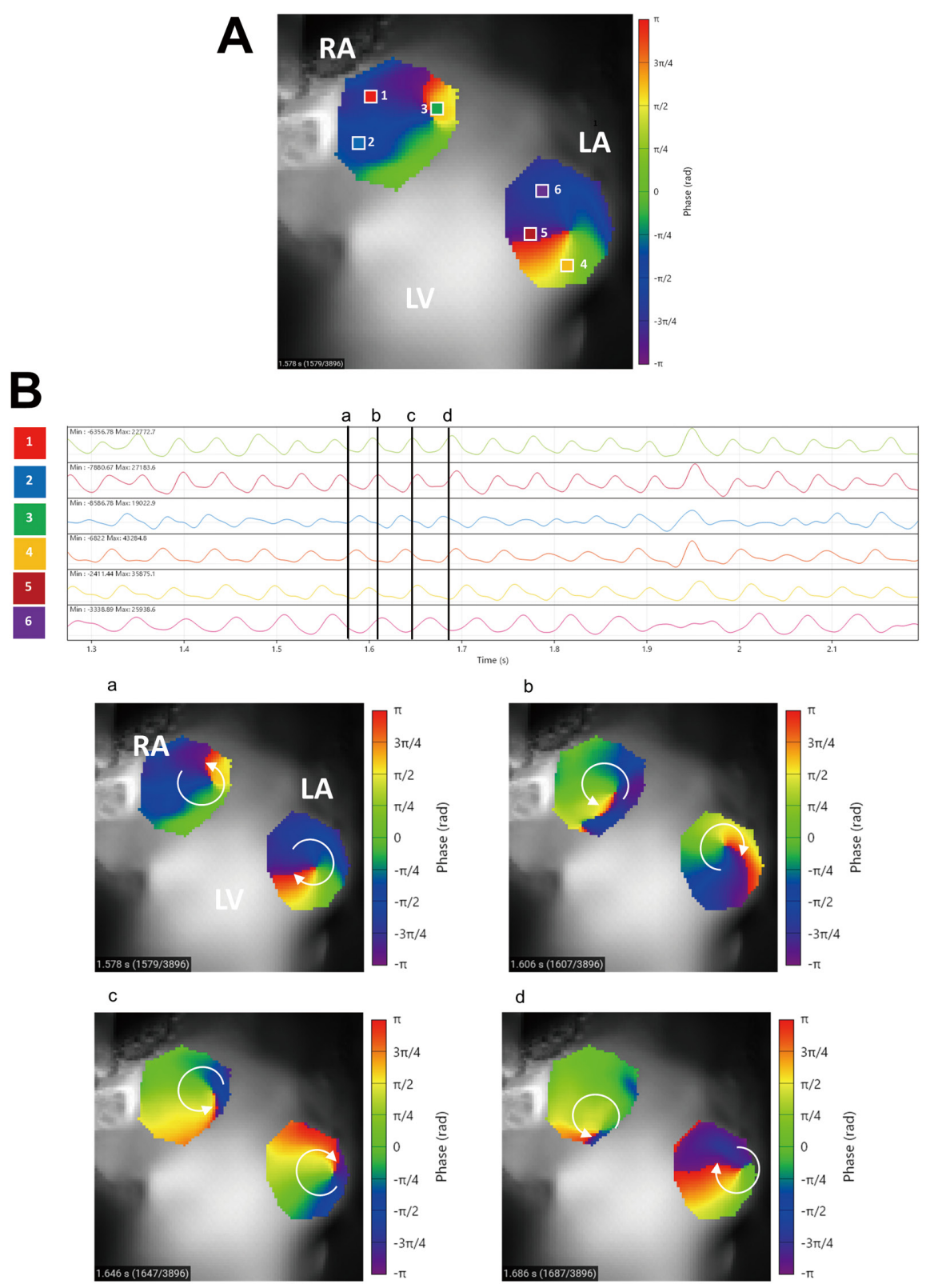

Figure 4. (A) Optical phase map of short-duration spiral atrial fibrillation (AF) in wild-type (WT) mice. The 2 vortices of both atria are rotating in opposite directions. Points 1-3 were in the right atrium (RA) and points 4-6 were in the left atrium (LA). Two spiral waves were present in the RA and LA independently. (B) Optical action potentials of points 1-6 are shown in the upper part of the figure. A phase map for $\mathbf{a}-\mathbf{d}$ is shown in the lower part of the figure. The phase map showed a counterclockwise rotation in the RA, and a clockwise rotation in the LA (Supplementary Movie).

wise in the LA (Supplementary Movie). Furthermore, we observed spiral type AF with meandering phase singularity (Figure 5, Supplementary Movie). The phase singularity of spiral AF was on the posterior side of the RA for the first part. The core gradually moved to the upper right of the RA and it stabilized at the right-hand side center of the RA. Finally, AF terminated in $40 \mathrm{~s}$.

This atrial analysis during various types of AF also suggested that an antegrade perfusion is sufficient for investigating various types of AA using optical mapping.

\section{Creation of an Artificial Atrial Substrate}

All mechanisms of short-duration AF were non-stationary and spontaneously terminated in short duration. The common point of these mechanisms of short-duration AF were instability of core and wave propagation. To create an artificial AA substrate, we used laser irradiation under an antegrade perfusion method. We could control laser-irradiated tissue damage by using a low-power laser, artificial substrate shape, and size by adjusting the laser system (Figure 6A). We determined a particular shape for the arti- 


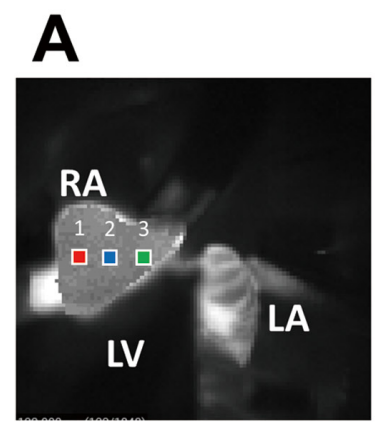

\section{B}
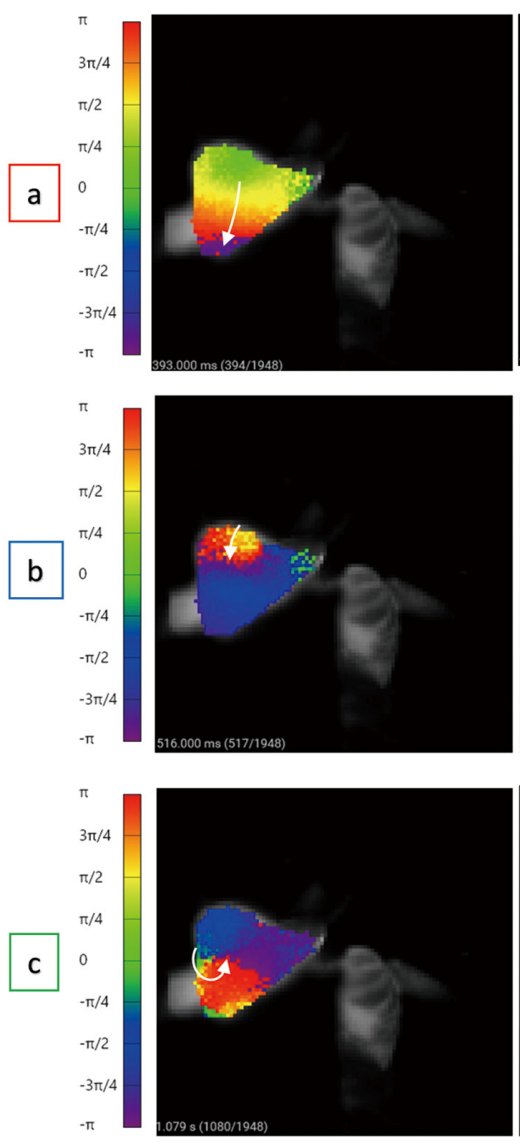
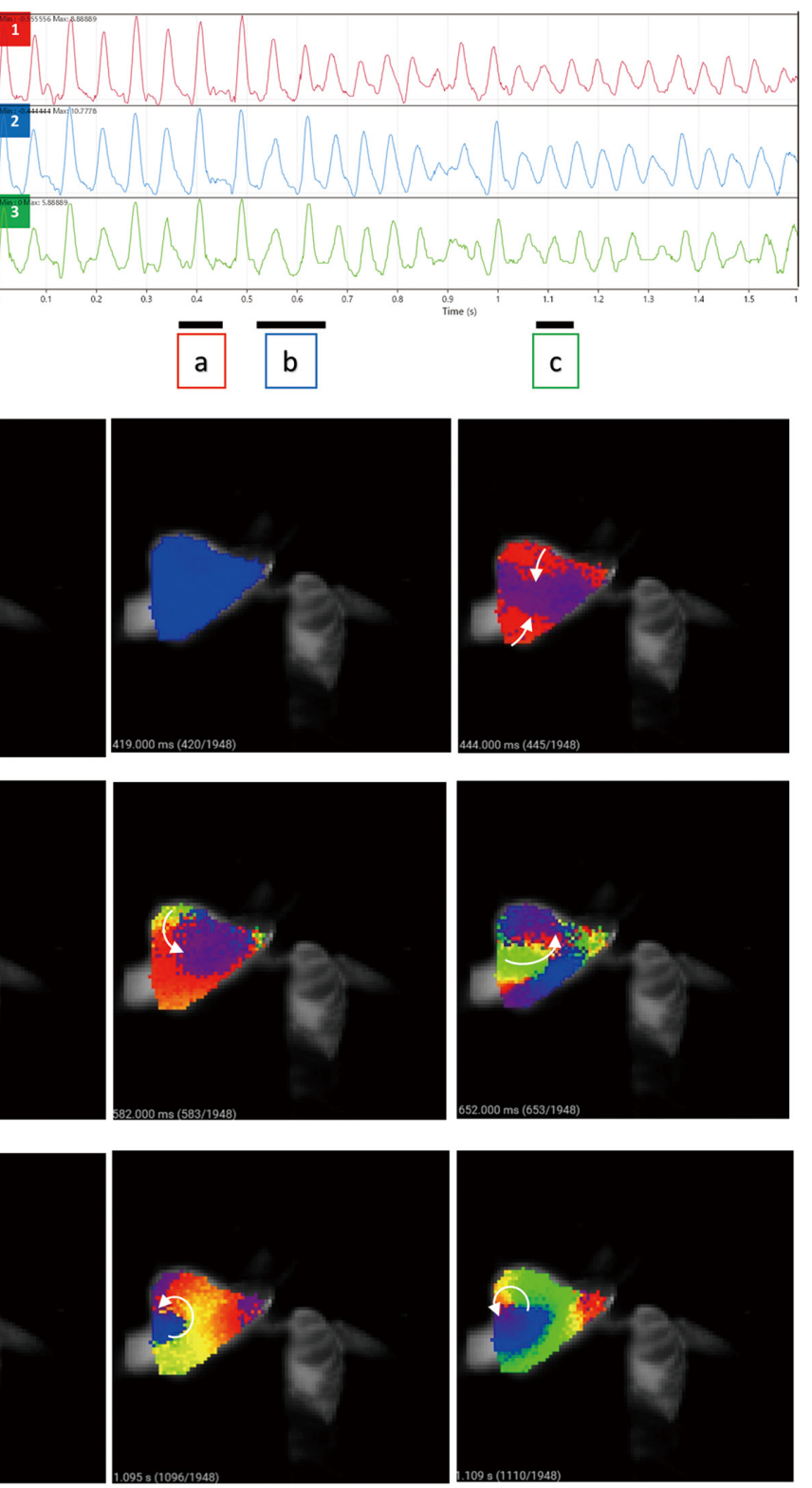

Figure 5. Drifting of spiral atrial fibrillation $(A F)$ in the right atrium (RA). (A) Optical action potentials of the RA in points 1-3 are shown in the right panel. Initially, action potential showed a comparatively long cycle length until phase $\mathbf{a}$. Action potential altered in phase b. Cycle length was shortened and action potential fluctuated in phase c. (B) Phase map during drifting spiral AF was shown (Supplementary Movie). In phase a, excitement is coming from the roof and bottom of the RA. The center of the spiral AF is presumed to be behind the RA. In phase $\mathbf{b}$, the center of the spiral AF moved to the right superior site of the RA. In phase $\mathbf{c}$, the center of the spiral AF settled into the right middle of the RA.

ficial atrial substrate with reference to self-sustained reentry circuits in a computer simulation, ${ }^{\mathbf{2 4}, 25}$ and we added a wedge-shaped dent to circle the substrate. Laser irradiation was performed on either the RA $(n=4)$ or the LA $(n=4)$. We conducted this experiment with a non-laser group (group 1: control, $n=8$ ), a laser-irradiated circular substrate group (group 2: circular-shape group, $n=8$ ), and a laser-irradiated circular substrate with wedge-shaped dent group (group 3: wedge-shaped dent group, $n=8$ ). In this study, long-lasting AA was defined being $>30 \mathrm{~min}$. In some cases, AA lasted $>60 \mathrm{~min}$ (Figure 6B). Atrial arrhythmia that lasted for $>65-70 \mathrm{~min}$ was discontinued due to the limited time available in the laboratory. Long-lasting AA was not induced in group 1 and group 2, but greatly induced in group $3(6 / 8,75 \%)$ (Figure 6C). The duration of AA was significantly extended in group $3(44.0 \pm 24.6 \mathrm{~min}$, 


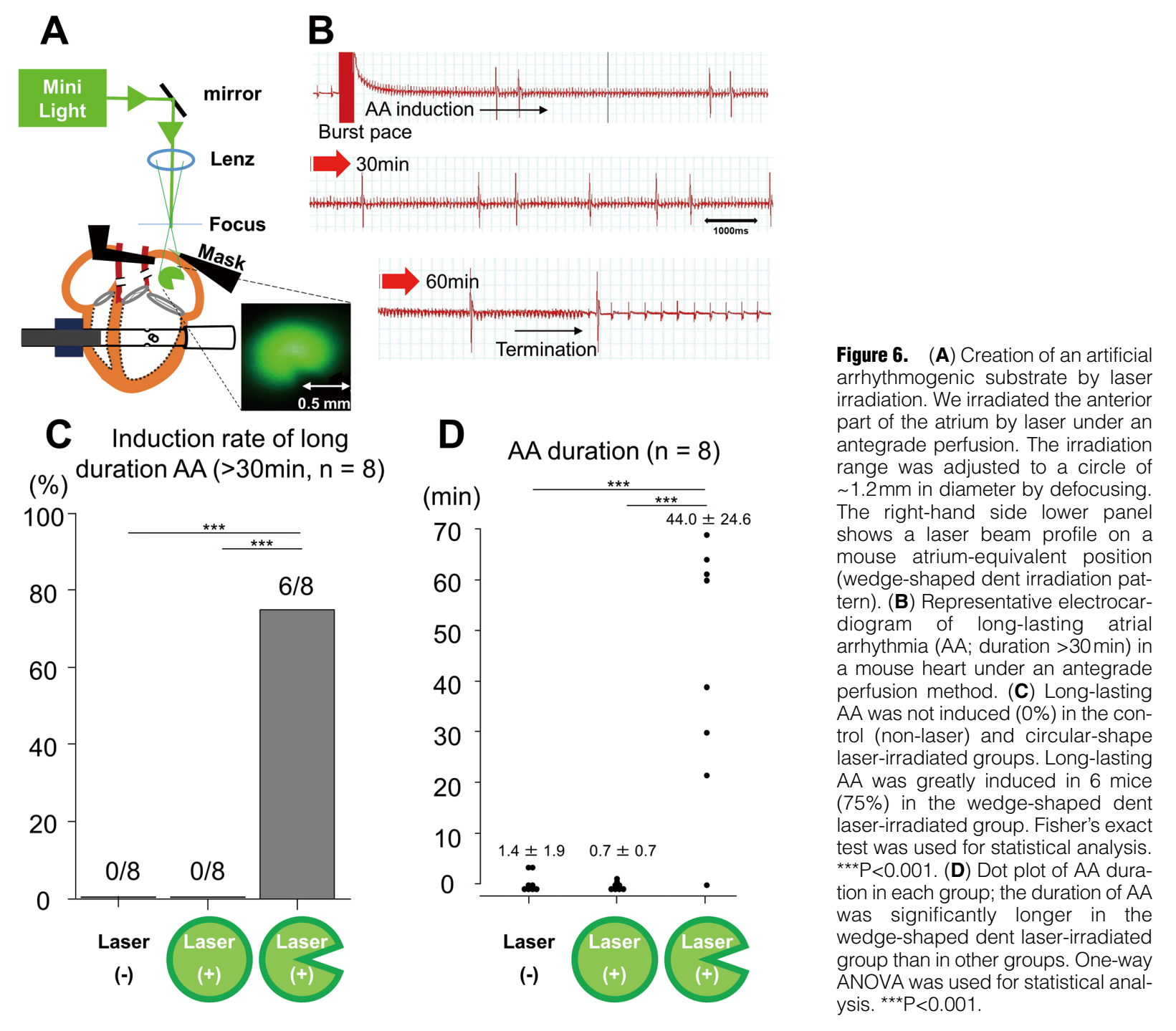

$\mathrm{n}=8)$ compared to group $1(1.4 \pm 1.9 \mathrm{~min}, \mathrm{n}=8$, vs. group 3, $\mathrm{P}<0.001)$ and group $2(0.7 \pm 0.7 \mathrm{~min}, \mathrm{n}=8$, vs. group 3 , $\mathrm{P}<0.001$ ) (Figure 6D). Thus, the artificial arrhythmia substrate we created successfully enabled AA to persist.

\section{Difference in Electrophysiological Mechanism Between Short-Duration AF and Sustained AT}

We observed the optical action potentials in wedge-shaped dent irradiated hearts to confirm the mechanism of longlasting AA and compared electrophysiological features with short duration spiral AF.

The phase singularity of spiral AF exhibited a non-stationary, meandering trajectory. Trajectories of phase singularity (x-y-t plots) of spiral AF (LA of Figure 4) are shown in Figure 7A. The spiral AF terminated after a short duration.

The phase map of long-lasting AA in the wedge-shaped dent group was stably rotating counterclockwise around the laser irradiation site (Supplementary Movie). The cycle length of AA was almost constant at approximately 65-75 $\mathrm{ms}$ and this arrhythmia resembled to reentrant AT. We plotted the phase singularity of long-lasting AA with white spots and created a space-time (x-y-t) graph (Figure 7B). The trajectory of phase singularity showed constant movement. These findings indicated that we successfully generated the artificial atrial substrate of a stationary and sustained reentry-like AT.

\section{Tissure Damage on Myocardium by Low-Power Laser}

We used a low-power laser and there was no apparent damage to the atrial surface due to irradiation. More than moderate power and focused laser irradiation can make a perforation on the atrial surface. Hematoxylin and eosin (HE) staining of laser-irradiated myocardial sections revealed no histological changes (Figure 8A). To confirm the slight damage made by low-power laser irradiation, we used autofluorescence to observe the NADH level after laser irradiation. The atrial NADH level is partially uneven because of the existence of atrial pectinate muscle. On atrium NADH assessment, a detailed morphological change and slight tissue damage caused by laser irradiation could not be accurately identified. We used the left ventricular surface to confirm the morphology and tissue damage caused by laser irradiation instead of atrium. A decrease in 

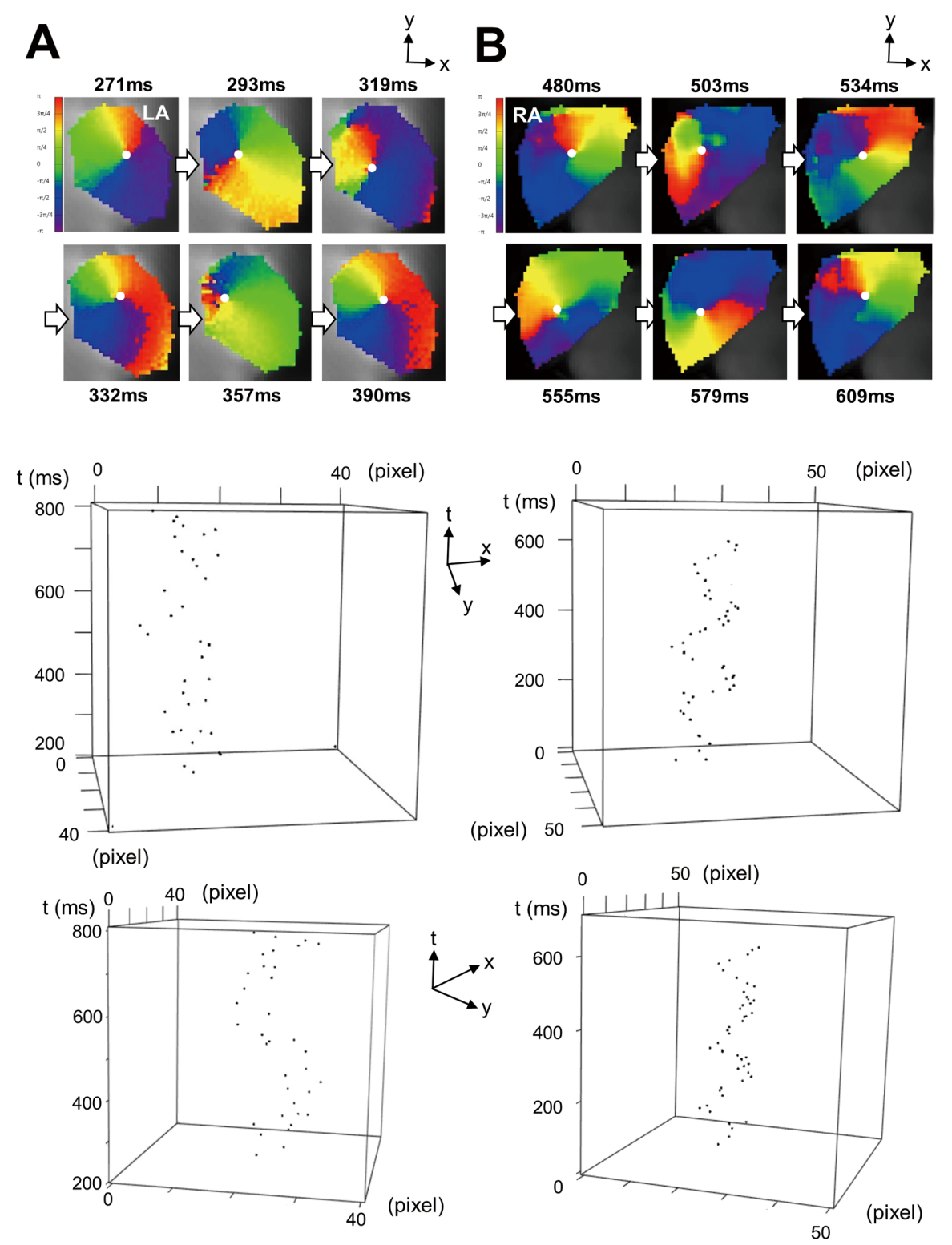

Figure 7. (A) Optical phase map and phase singularity space-time plots of short-duration spiral atrial fibrillation (AF) in wild-type (WT) mice (analysis of the left atrium (LA) in Figure 4). The singularity of the phase map was plotted with white spots in the upper panel. The singularity of the phase map was plotted with black dots in the middle and lower panels. It showed meandering with time. (B) Optical phase map and phase singularity space-time plots of long-lasting atrial arrhythmia (AA) post wedge-shaped dent laser irradiation in the right atrium (RA). The phase singularity drew almost the same trajectory rotating counterclockwise around the regularly laser irradiated area (Supplementary Movie).

NADH was observed in the irradiation area. The boundary between the irradiation area and non-irradiation area was obvious (Figure 8B,C). In the case of wedge-shaped dent irradiation, we observed that the NADH level was maintained in the wedge-shaped portion (dent) shielded by the mask. We confirmed that the circular-shape and wedge-shaped dent tissue damage on myocardium was created. Decreased NADH indicates laser irradiationinduced atrial tissue damage, and the clear morphology of reduced NADH indicates that the substrate for artificial AA was successfully produced as we intended.

\section{Discussion}

Mice have small atria to investigate AA. In classical Langendorff perfusion, atria tend to be collapsed. Making an artificial substrate on the atrium by using laser irradiation from the front using the classical Langendorff perfusion method was technically difficult to achieve. Therefore, we established a novel antegrade perfusion from the LV to make the atrium a little expanded. Both atria were a little expanded in an antegrade perfusion method (Supplementary Figure $\mathbf{C}-\mathbf{E}$ ). LA expansion was most likely due to mitral 
A

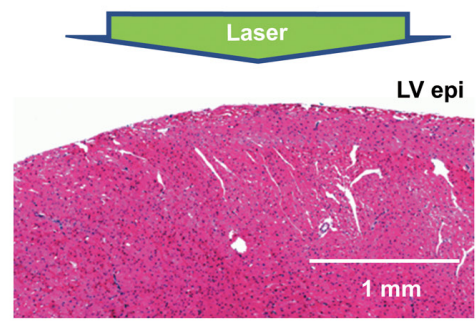

B

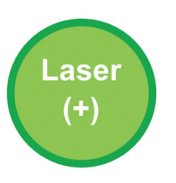

NADH fluorescence

Intensity distribution
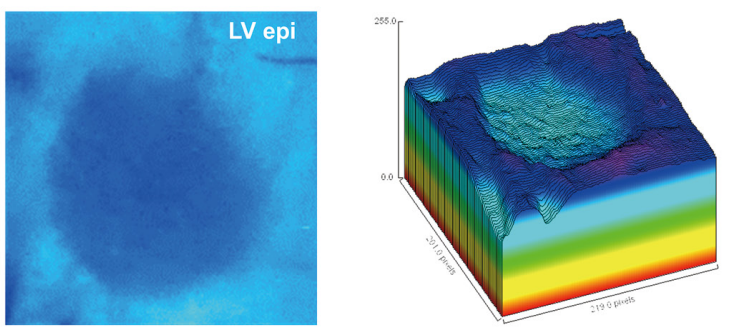

C
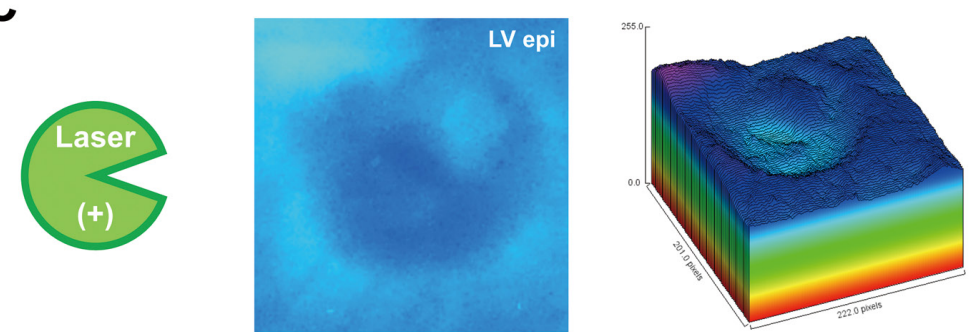

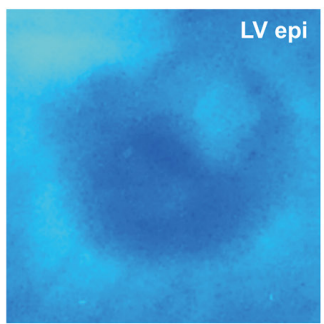

Figure 8. (A) Hematoxylin and eosin (HE) staining of the ventricular surface. Low energy laser irradiation did not cause histological changes in the myocardium. Atrial muscle was thin and difficult to evaluate for histological change. Left ventricular muscle was used for histological evaluation. Epi, epicardium. (B,C) Nicotinamide adenine dinucleotide (NADH) fluorescence images on the myocardium (left ventricular (LV) epicardium) after laser irradiation. NADH fluorescence of the myocardium after laser irradiation is shown in the middle panel. Three-dimensionally constructed intensity distribution of NADH autofluorescence is shown in the right panel. In circular-shape irradiation, a clear circular decrease in NADH was observed. (B) In wedge-shaped dent irradiation, a clear circular decrease with a dent in NADH was observed $(\mathbf{C}) .1$ pixel $=0.01 \mathrm{~mm}$.

regurgitation caused by LV perfusion and preservation of bilateral superior lungs. The dilation of the RA was thought to be due to the coronary venous flow through the coronary arteries and limited leakage by SVC clamping. Both the LA and RA pressure were maintained at a low level without non-physiological high pressure (Figure 2A). A slight expansion of both atria under an antegrade perfusion make it possible to create an artificial substrate on the atrium. This method also enables atrial electrical characteristics to be assessed. In optical mapping, conduction velocity and action potential duration (Figure 2B) were not different from past reports, and normal electrical propagation from the SN to the RA and LA was observed (Figure 2C,D). However, second degree $(3 / 8,38 \%)$ or complete AV block $(2 / 8,25 \%)$ were often observed (Figure 2E). The cause of AV block was speculated by AV node or His bundle injury because of a septal puncture caused by the catheter. Optical mapping of the ventricle was often difficult using this method during AV block, but was possible during ventricular pacing. The presence of an AV block was suitable for observing AA because it eliminates motion artifacts due to ventricular contraction. Our novel method for AA assessment was technically easier to set up compared with the traditional Langendorff perfusion.

We attempted to induce AF by using burst pacing with bipolar electrodes pressed directly on the front surface of the RA or LA. However, the induction method often failed to induce AF under an antegrade perfusion method (Figure 3A). We changed the stimulation locations to the posterior LA between the left and right hilar margin, which were close to the ostium of PVs (red and yellow electrodes in Figure 1D). This PV burst pacing $(30 \mathrm{~V}, 50 \mathrm{~Hz}, 500 \mathrm{~ms})$ successfully induced short-duration AF. The high arrhythmogenesis of PV and the large area of bipolar pacing might be effective for constant AF induction. Various electrical characteristics reported in the past were observed during short-duration AF. Focal and spiral type AF were observed (Figures 3C-E, 4,5). Common points of shortduration AF mechanisms were shorter cycle length and the meandering phase singularity of AF. It disappeared while continuously changing. Small murine hearts have lowgrade atrial arrhythmogenesis. ${ }^{18}$ We hypothesized that if we succeed in creating an atrial substrate that sustains AA in mice, the substrate must be one of the important keys of atrial arrhythmogenesis. Although we had tried to create such a substrate by heating and freezing in preliminary experiment, we failed to make such a substrate due to uncontrollable factors such as severity of tissue damage, size, and shape. To control for these substrate factors, we used laser irradiation. Unlike our initial expectation, a circular-shape substrate did not sustain AF. In contrast, the duration of AF tended to be shorter (Group 1: $1.4 \pm 1.9 \mathrm{~min}, \mathrm{n}=8$ vs. Group 2: $0.7 \pm 0.7 \mathrm{~min}, \mathrm{n}=8$, n.s.), as if AF ablation was performed (Figure 6D). When a small wedge-shaped dent was added to the circle, we succeeded in achieving sustained AT induction. This result implies that a seemingly circular-shape substrate with minor extrinsic changes causes reentrant arrhythmias. Atrial remodeling caused by inflammation, ischemia, and pressure overload in hypertension, and a failing heart would be 
able to create small substrate changes that sustain AA; however, the substrate created by laser has a clear edge and may be different from those seen as a result of atrial remodeling. Our murine experimental AT model analyzed by optical mapping (Figure 7B, Supplementary Movie) might resemble reentrant AT due to incomplete PV isolation in humans. ${ }^{26} \mathrm{We}$ could not create the substrate that progressed from paroxysmal to persistent $\mathrm{AF}$, but our study might have proved one of the mechanisms of reentrant AT due to incomplete ablation. The STAR AF II trial $^{6}$ could not show the effectiveness of additional linear ablation; one of the causes was speculated to be incomplete linear ablation. Our experimental result would support the importance of a complete isolation line to prevent recurrence of reentrant $\mathrm{AT}$ during $\mathrm{AF}$ ablation.

The substrate we created has another special feature; the reentry circuit was small, only $1.2 \mathrm{~mm}$ in diameter. Although the action potential and refractory period in the murine heart were short, such a small-size reentry was impressive. ${ }^{18}$ A sustained reentrant circuit may be close in concept to the self-sustained reentrant circuit proposed by a mathematical model, ${ }^{23}$ and the substrate of wedgeshaped dent laser irradiation behaved as a stationary rotor core similar to a self-sustained reentrant circuit and generated radical electrical waves continuously.

A limitation of this study is that the experiments were performed ex vivo. Laser energy is absorbed by red blood cells, so it is expected that to create an arrhythmogenic substrate in vivo would be difficult. Conducting the experiments on a small animal is another limitation. We will have to examine this phenomenon in larger animals in the future.

\section{Sources of Funding}

This work was supported by JSPS KAKENHI (Grant Number JP20K08461).

\section{Disclosures}

T.A. is an Editorial Board member of Circulation Journal.

\section{IRB Information}

The present study was approved by The Animal Care and Use Committee (National Defense Medical College). Reference number: 15051.

\section{Data Availability}

The data underlying this article are available in the article and in the Supplementary material online.

\section{References}

1. Miyasaka Y, Barnes ME, Gersh BJ, Cha SS, Bailey KR, Abhayaratna WP, et al. Secular trends in incidence of atrial fibrillation in Olmsted county, Minnesota, 1980 to 2000, and implications on the projections for future prevalence. Circulation 2006; 114: 119-125.

2. Iwasaki Y, Nishida K, Kato T, Nattel S. Atrial fibrillation pathophysiology, implications for management. Circulation 2011; 124: $2264-2274$.

3. John Camm A, Lip GYH, De Caterina R, Savelieva I, Ata D, Hohnloser SH, et al. 2012 focused update of the ESC guidelines for the management of atrial fibrillation: an update of the 2010 ESC guidelines for the management of atrial fibrillation. Developed with the special contribution of the European Heart Rhythm Association. Eur Heart J 2012; 33: 2719-2747.

4. Steinberg BA, Hellkamp AS, Lokhnygina Y, Patel MR, Hankey GJ, Becker RC, et al. Higher risk of death and stroke in patients with persistent vs. paroxysmal atrial fibrillation: Results from the ROCKET-AF Trial. Eur Heart J 2015; 36: 288-296.

5. Nattel S, Guasch E, Savelieva I, Cosio FG, Valverde I, Halperin $\mathrm{JL}$, et al. Early management of atrial fibrillation to prevent cardiovascular complications. Eur Heart J 2014; 35: 1448-1456.
6. Verma A, Jiang CY, Betts TR, Chen J, Deisenhofer I, Mantovan $\mathrm{R}$, et al. Approaches to catheter ablation for persistent atrial fibrillation. New Engl J Med 2015; 372: 1812-1822.

7. Schrickel JW, Bielik H, Yang A, Schimpf R, Shlevkov N, Burkhardt $\mathrm{D}$, et al. Induction of atrial fibrillation in mice by rapid transesophageal atrial pacing. Basic Res Cardiol 2002; 97: $452-460$.

8. Ozcan C, Battaglia E, Young R, Suzuki G. LKB1 knockout mouse develops spontaneous atrial fibrillation and provides mechanistic insights into human disease process. $J$ Am Heart Assoc 2015; 4: $\mathrm{e} 001733$.

9. Li N, Csepe TA, Hansen BJ, Sul LV, Kalyanasundaram A, Zakharkin SO, et al. Adenosine-induced atrial fibrillation: Localized reentrant drivers in lateral right atria due to heterogeneous expression of adenosine A1 receptors and GIRK4 subunits in the human heart. Circulation 2016; 134: 486-498.

10. Wan E, Abrams J, Weinberg RL, Katchman AN, Bayne J, Zakharov SI, et al. Aberrant sodium influx causes cardiomyopathy and atrial fibrillation in mice. J Clin Invest 2016; 126: 112-122.

11. Xu J, Cui G, Esmailian F, Plunkett M. Atrial extracellular matrix remodeling and the maintenance of atrial fibrillation. Circulation 2004; 109: 363-368.

12. De Vos CB, Pisters R, Nieuwlaat R, Prins MH, Tieleman RG, Coelen RJS, et al. Progression from paroxysmal to persistent atrial fibrillation clinical correlates and prognosis. $\mathrm{J} \mathrm{Am} \mathrm{Coll}$ Cardiol 2010; 55: 725-731.

13. Burstein B, Nattel S. Atrial fibrosis: Mechanisms and clinical relevance in atrial fibrillation. $J$ Am Coll Cardiol 2008; 51: 802-809.

14. Voigt N, Trausch A, Knaut M, Matschke K, Ravens U, Dobrev D. Left-to-right atrial inward rectifier potassium current gradients in patients with paroxysmal versus chronic atrial fibrillation. Circ Arrhythm Electrophysiol 2010; 5: 472-480.

15. Caballero R, Harm BP, Gonza M, Osuna L, Almendral J, Atienza F, et al. In humans, chronic atrial fibrillation decreases the transient outward current and ultrarapid component of the delayed rectifier current differentially on each atria and increases the slow component of the delayed rectifier current in both. $J \mathrm{Am}$ Coll Cardiol 2010; 55: 2346-2354.

16. Martins RP, Kaur K, Hwang E, Ramirez RJ, Willis BC, FilgueirasRama D, et al. Dominant frequency increase rate predicts transition from paroxysmal to long-term persistent atrial fibrillation. Circulation 2014; 129: 1472-1482.

17. Ackers-johnson M, Li PY, Holmes AP, Brien SO, Pavlovic D, Foo RS. New methods in cardiovascular biology: A simplified Langendorff-free method for concomitant isolation of viable cardiac myocytes and nonmyocytes from the adult mouse heart. Circ Res 2016; 119: 909-920.

18. Huang CLH. Murine electrophysiological models of cardiac arrhythmogenesis. Physiol Rev 2017; 97: 283-409.

19. Sill B, Hammer PE, Cowan DB. Optical mapping of Langendorffperfused rat hearts. $J$ Vis Exp 2009; 30: 1138

20. Coremans JMCC, Ince C, Bruining HA, Puppels GJ. (Semi-) quantitative analysis of reduced nicotinamide adenine dinucleotide fluorescence images of blood-perfused rat heart. Biophys $J$ 1997; 72: $1849-1860$.

21. Asfour H, Wengrowski AM, Jaimes 3rd R, Swift LM, Kay MW. $\mathrm{NADH}$ fluorescence imaging of isolated biventricular working rabbit hearts. J Vis Exp 2012; 65: 4115.

22. Mayevsky A, Rogatsky GG. Mitochondrial function in vivo evaluated by NADH fluorescence: From animal models to human studies. Am J Physiol, Cell Physiol 2007; 292: C615-C640.

23. Nattel S. New ideas about atrial fibrillation 50 years on. Nature 2002; 415: 219-226.

24. Zykov V, Krekhov A, Bodenschatz E. Fast propagation regions cause self-sustained reentry in excitable media. Proc Natl Acad Sci USA 2017; 114: 1281-1286.

25. Alonso S, Bär M. Reentry produced by small-scale heterogeneities in a discrete model of cardiac tissue. J Phys Conf Ser 2016; 727: 012002.

26. Chun KRJ, Bansch D, Ernst S, Ujeyl A, Huang H, Chu H, et al. Pulmonary vein conduction is the major finding in patients with atrial tachyarrhythmias after intraoperative maze ablation. $J$ Cardiovasc Electrophysiol 2007; 18: 358-363.

\section{Supplementary Files}

Supplementary Movie. Optical phase map movies in Figure 4, Figure 5B, and Figure 7.

Please find supplementary file(s);

http://dx.doi.org/10.1253/circj.CJ-20-1286 